global human spaceflight infrastructure from which science can only benefit an international Moon base being the obvious next step.

Ian Crawford

School of Earth Sciences, Birkbeck College,

Malet Street, London WC1E 7HX, UK

\section{Illustration: database pictures tell a true story}

Sir - Julio M. Ottino in his Commentary "Is a picture worth 1,000 words?" (Nature 421, 474-476; 2003) divides images into two categories: those that convey data and those that illustrate scientific ideas. He defends the practice of image manipulation as sometimes being a necessary part of the process of discovery, yet expresses concern about the blurring of the line between fantasy and reality in scientific illustration.

It may be that the two categories are not that distinct. The European Space Agency's programme Innovative Technologies from Science Fiction for Space Applications assumes that even the most fantastic illustrations may be a useful stimulus to science. On the other hand, images that purport to convey factual data may convey something else entirely. Many observers, for example, were convinced that they could see little human beings in the sperm images produced by the sixteenth-century microscopist Antoni van Leeuwenhoek, and they recorded this observation as fact, presumably influenced by their beliefs.

Today, we can and should capture not just an image but information documenting the process of image creation itself, from the original unmodified data to the final web-ready or journal-ready artwork. If questions arise about the interpretation of an image, we need to be able to go back to the raw data or, if this is impossible, at least to have a full record of what was done to it, and why. For example, in performing video-enhanced contrast microscopy, one always subtracts a digital background 'mottle' image from the live video stream to obtain the mottle-free video frames that are recorded, and viewers need to be informed that background subtraction has been carried out. Similar arguments relate to the point spread functions used in preparing the deconvolved fluorescence images mentioned in the article.

Of course, as in the days of pen-and-ink illustration, scientists should still consider the purpose served by each image in their publications, and should make these objectives clear to their readers. Scientists today have the additional responsibility of recording the processes by which images are created, so that these can be accurately replicated. Currently, such information is usually held only in the laboratory from which the image came, if it is recorded at all.

The BioImage Database Project (http://www.bioimage.org), part of ORIEL (Online Research Information Environment for the Life Sciences; http://www.oriel.org), will be a searchable database of multidimensional images of biological specimens. From its outset, we have felt it essential to acquire not just high-quality source images, but also the various images derived from them, and detailed metadata documenting the process of their creation. We believe that this approach addresses many of the issues raised by Ottino, and should be far more widely adopted.

Chris Catton, David Shotton

Image Bioinformatics Laboratory,

Department of Zoology, University of Oxford,

South Parks Road, Oxford OX1 3PS, UK

\section{Illustration: images fail to portray dynamic skies}

Sir - An example of the visual deception practised by scientific illustrators seeking greater impact - as described by Julio M. Ottino in his Commentary (Nature 421, $474 ; 2003)$ - is the increasingly prevalent practice of adding foreground stars and background galaxies to images generated from numerical simulations of galactic collisions. Examples appear in National Geographic 203, 2, 2003; and on the Gadget website at www.mpa-garching. mpg.de/gadget. Another is one of my simulations, published unadulterated on the cover of Nature on 9 March 1989, which was later "improved" by the imagemakers at NASA's Space Telescope Science Institute (http://hubblesite.org/ newscenter/archive/2001/22/video/c) without my consent.

Although one may agree that superimposed stars and galaxies add a bit of visual interest to the blankness of cyberspace, it is disturbing that these additions are entirely static. In reality, foreground stars hurtle past like snowflakes in a blizzard, and even background galaxies change noticeably over the hundreds of millions of years that are represented in the computer simulations. Static foreground stars and background galaxies undermine the basic idea of a dynamic Universe that these images and animations presumably attempt to convey.

Joshua E. Barnes

Institute for Astronomy, University of Hawaii, Honolulu, Hawaii 96822, USA

\section{Swift publication would reward good reviewers}

Sir - I completely agree with T. Clausen and O. B. Nielsen, who say in Correspondence (Nature 421, 689; 2003) that peer-reviewing needs to be adequately rewarded for the system to work efficiently. However, their proposed remedies, such as mandatory inclusion of reviews in CVs, do not seem promising, not least because this is already routinely done and does not seem to change the general picture.

While toiling on my considerable backlog of manuscripts to review, I came up with a scheme that might stand a better chance of improving the situation: diligent reviewing could be rewarded by speeding up publication of the reviewer's own research.

Currently, most of the prominent journals impose a strict deadline (typically between 7 and 14 days) on reviewers, yet the level of compliance is dismally low, as any author waiting for longer than two months for reviews of a submitted manuscript can testify.

My suggestion is that a researcher who meets the deadline for a particular journal with satisfactory reviews on, say, six consecutive occasions within a two-year period is guaranteed by the journal that his or her next submission will be given privileged status and reviewed within that same deadline. If the journal's editor cannot receive reviews of a privileged submission before the deadline, the journal would have to make a decision based on the other referees' comments or, if none had been received, accept the paper as it stands.

A possible variant is for journals that reject many submissions without review to guarantee to review a privileged submission (again, within the deadline). This is unlikely to burden these highprofile journals with piles of junk, because an individual writing six or more useful reviews for one of them within a limited time span is, by definition, a highly competent researcher.

Of course, a crucial aspect is the notion of a 'useful' review: to earn a privileged submission, reviews would have to be of high quality, as judged by the journal's editors, rather than one-liners. I believe that such direct feedback between a researcher's own publications and reviewing activity could seriously improve the peer-review system.

Feedback loops do wonders in biological systems; they just might work for science, too. Health, Bethesda, Maryland 20894, USA 